\title{
No imagination effect on boundary extension
}

\author{
${\text { Margaret P. } \text { Munger }^{1} \cdot \text { Kristi S. Multhaup }}^{1}$
}

Published online: 7 August 2015

(C) Psychonomic Society, Inc. 2015

\begin{abstract}
Boundary extension (BE) occurs when people falsely remember perceiving beyond the edges of a presented scene. Theorists argue that BE occurs because people mistakenly attribute information they have generated to the study stimulus - that is, they make a source memory error. Inspired by this idea, in six experiments we tested whether scene details resulting from explicit imagination would be misremembered as actual visual perceptions, resulting in increased $\mathrm{BE}$ as compared with standard instructions. In four experiments, undergraduates completed a BE task with separate study and test blocks; in two further experiments, undergraduates completed a trial-by-trial BE task $(N=290)$. Half of the participants elaborated on the study pictures (imagined smells and sounds, or what was to the left and right of the scene, or what a photographer would see by zooming in or out). Robust BE was found in all experiments, but none of the elaborations modified the size of $\mathrm{BE}$; therefore, $\mathrm{BE}$ is not to be affected by explicit elaboration and may be related to spatial rather than visual imagery ability.
\end{abstract}

Keywords Boundary extension · Source memory · Spatial imagery

In the present research, we investigated boundary extension (BE) as a type of source memory error. BE occurs when viewers falsely remember seeing a wider angle of a scene than they had actually studied (e.g., Hubbard, Hutchison, \&

Margaret P. Munger

mamunger@davidson.edu

Kristi S. Multhaup

krmulthaup@davidson.edu

1 Department of Psychology, Davidson College, Davidson, NC 28035, USA
Courtney, 2010). Source memory is the ability to keep track of where information came from (e.g., Johnson, Hashtroudi, \& Lindsay, 1993; Lindsay, 2008). For example, sometimes people obtain information from the world, as from a photo, and sometimes they generate it themselves, as when they imagine a scene. These two literatures come together in the multisource view of scene representation (Intraub, 2010; Intraub \& Dickinson, 2008), which highlights that individuals can utilize information about scenes from a variety of sources, including visual perception, amodal continuation of surfaces, world knowledge of scene classification, and contextual associations. (Intraub and Dickinson, 2008; see also Intraub, 2010, 2012) argued that BE occurs because people misattribute scene-relevant information that they have generated internally while viewing a picture (or after viewing it) to the picture itself. In short, the argument is that $\mathrm{BE}$ is a form of source memory error.

Although BE is often studied with visual stimuli, BE also has been observed following blindfolded, haptic explorations of tabletop scenes (Intraub, 2004). Specifically, when participants used both hands to explore a tabletop scene involving three to five objects and were then asked to adjust a surrounding wooden frame, they made the frame larger than the original (Intraub, 2004). The act of physically exploring the objects with their hands and then adjusting the frame led to BE, highlighting the cross-modal aspect of multisource scene representation (Intraub, 2010, 2012). Both visual and haptic exploration have shown BE, which Intraub $(2010,2012)$ sees as evidence that multiple sources of information are used to create a larger representation of the scene.

In the present research, we assessed whether participants' imagination of sensory details affects BE, since this manipulation affects other source memory errors. Thomas, Bulevich, and Loftus (2003), for example, demonstrated that the elaboration of sensory details during study increases source 
memory errors at test. More specifically, Thomas et al. asked participants to identify which simple actions they had actually performed at study and which actions they had only imagined. As compared with participants who engaged in simple imagining (e.g., Imagine kissing the frog.), participants who engaged in elaborate imagining (e.g., Imagine kissing the frog. Imagine the color of the frog. Imagine the feel of the frog against your lips.) showed increased false "did" responses, incorrectly claiming they had performed actions they had elaborately imagined. This was true when the visual and sensory details were provided by the researchers, and when the details were participant-generated. In the present series of experiments, we asked participants to view photographs and elaborate on scenes cross-modally (what smells and sounds would occur, Exps. 1a-2b) or visually (what was to the left and right of the picture, Exp. 3a; what the photographer would see by zooming in or out, Exp. 3b). We expected the elaboration conditions to lead to richer scene representations, and more BE, in parallel with the elaborate imagination conditions leading to increased source memory errors for actions (Thomas et al. 2003).

Prior evidence had suggested that instructions to imagine additional spatial information can influence BE (Intraub, Gottesman, \& Bills, 1998). Participants shown outlines of central objects and instructed to imagine specific spatial backgrounds exhibited $\mathrm{BE}$, whereas those instructed to imagine object color did not. In addition, more BE was observed when participants were shown line drawings than when they were shown photographs (Gagnier \& Intraub, 2012). Gagnier and Intraub's results suggest that photographs may be the best kind of stimuli to begin exploring enhancement of $\mathrm{BE}$, because line drawings could already produce BE at ceiling. Thus, in the present series of experiments we used photographs as the stimuli and asked participants to imagine additional sensory details. The typical scenes used in BE experiments tend to have fairly isolated, central objects, but to encourage rich elaborations, for five of the present experiments we used scenes with multiple items (see Fig. 1).

Notably, imagination per se does not always increase source memory errors (Foley, Foy, Schlemmer, \& BelserEhrlich, 2010). Using the Deese-Roediger-McDermott paradigm with participants asked to remember lists of pictures or words, Foley et al. (2010) found reduced errors when participants were instructed to deliberately generate images of pictures or words. They suggested that the act of generating the images distinguished these items from the actual list items, and thus reduced source memory errors. Foley (2012) highlighted a distinction between spontaneous imagery and intentional self-generated imagery; the present research included both, and we will return to this distinction in the General Discussion. To the extent that scene representation, as measure by $\mathrm{BE}$, is related to imagining isolated objects (the task in Foley et al. 2010), additional imagery instructions might reduce $\mathrm{BE}$.

Just as experimental manipulations have affected source memory performance, so individual-difference variables have as well. For example, Markham and Hynes (1993) compared the source memory performance of undergraduates who selfreported high vividness of visual imagery with that of counterparts who reported low vividness. The control participants saw geometric shapes and rated their complexity; the experimental participants imagined stimuli that were half shapes as being symmetrically completed around an axis. At test, all participants identified geometric forms as having been whole or half shapes during study. The participants with high selfreported vividness of visual imagery made source memory errors, confusing the imagined and perceived geometric shapes, more than the other groups did. Thus, these data suggest that people high in visual imagery may find it particularly difficult to discriminate between what they had generated and what had been presented to them (see also Finke, Johnson, \& Shyi, 1988).

In four of the present experiments, we assessed participants' visual imagery and correlated those scores with their BE scores. On the basis of Markham and Hynes (1993), one might expect that participants high in visual imagery would have more trouble than participants low in visual imagery in discriminating information they had generated from what had been presented to them, leading to a positive correlation between visual imagery ability and BE scores. In tentative support of this, Mullally, Intraub, and Maguire (2012) found that amnesic patients had lower scores than controls on a "scene probe" task that assessed their ability to imagine beyond the borders of a presented photograph. In addition, the amnesic patients had attenuated BE. Although the authors interpreted their results specifically in terms of scene structure, the results also suggest that a lack of imagery ability might impact other cognitive tasks. Specifically, for the present discussion, the findings that amnesics had lower imagining abilities and less BE than controls is correlational evidence that lack of ability to imagine beyond a scene's borders is associated with reduced BE. Stated another way, participants low in visual imagery may have low BE scores; that is, there may be a positive correlation between imagery and BE.

In the present experiments, a given picture could be shown as a close-up (C) or a wide-angle shot (W) at study and at test (e.g., $C W$ indicates close-up at study and wide-angle at test; $C C$ indicates close-up at both study and test-i.e., an identical view). For each experiment, there were three critical analyses, the first two involving replication of typical BE results, and the last asking whether instructions during the study block affected the BE results. First, how would participants rate identical pictures $(\mathrm{CC}, \mathrm{WW})$ ? Importantly, rating an identical photograph as being closer indicates $\mathrm{BE}$ for the original view. Previous work has revealed more BE (i.e., more extreme 
Fig. 1 Example photographs of cities, markets, deserts, and forests from the world set used in Experiments 1a, 1b, 2a, 3a, and 3b: (A) Close-up and (B) wideangle views
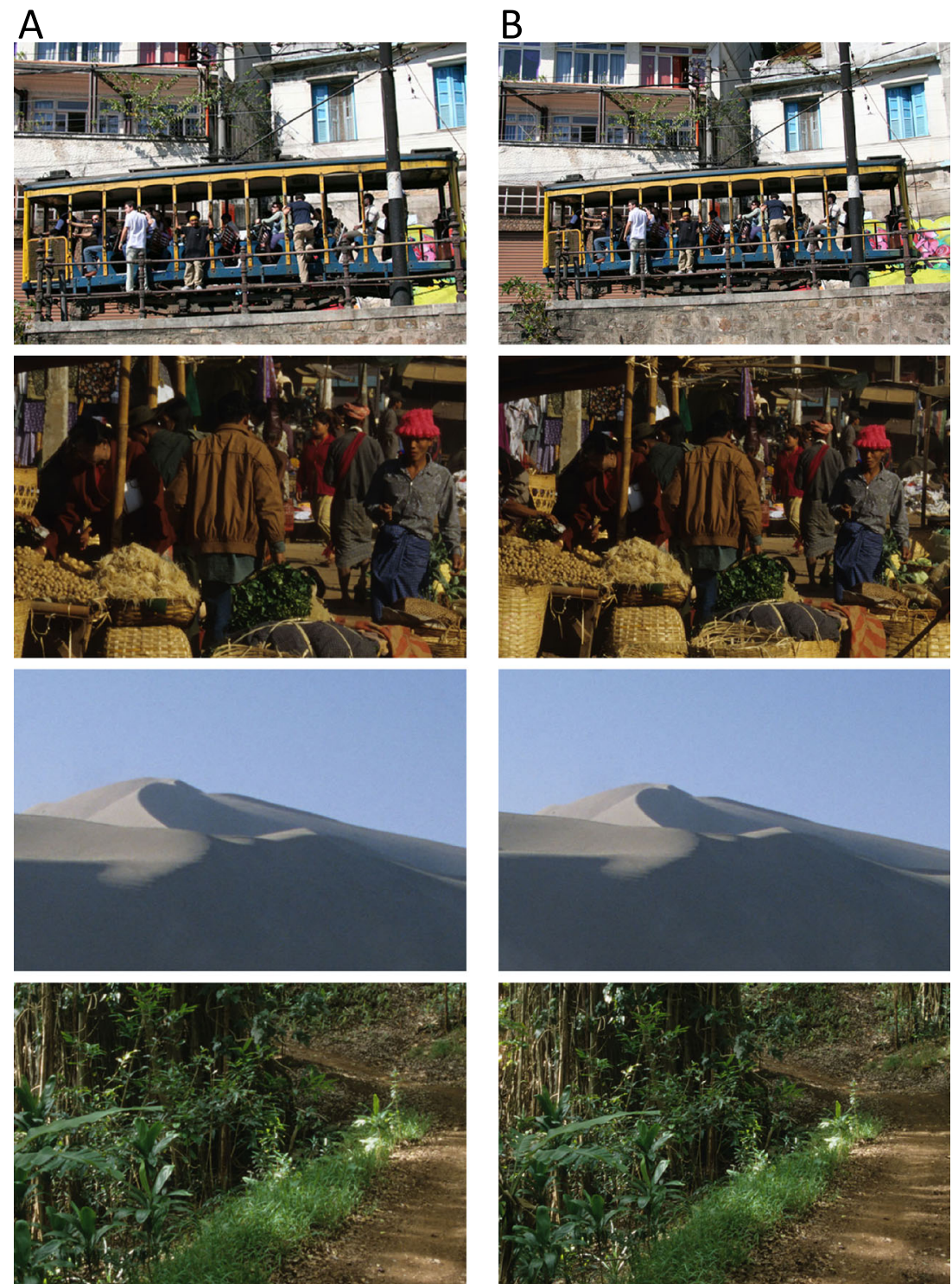

"closer" ratings) for close-up than for wide-angle views (e.g., Intraub \& Dickinson, 2008), but the most important pattern for both CC and WW is significant negative ("closer") ratings, even though the correct response is "the same." Second, would participants notice the "different" trials (CW, WC) equally, or would they mistake $\mathrm{CW}$ trials as being identical, indicating extension at study, while viewing $\mathrm{WC}$ as being quite different? Typical BE results show smaller ratings for $\mathrm{CW}$ than for WC trials, with $\mathrm{CW}$ trials sometimes showing positive rather than negative ratings (e.g., Intraub \& Dickinson, 2008). Finally, would the instruction set (elaboration vs. standard conditions) influence either the "same" trials $(\mathrm{CC}, \mathrm{WW})$ or "different" trials $(\mathrm{CW}, \mathrm{WC})$ ? To assess these three questions, we followed the analysis used in other $\mathrm{BE}$ work (e.g., Dickinson \& Intraub, 2008) and conducted two mixed-design analyses of variance (ANOVAs), 2 (Same Angles) $\times 2$ (Instruction Set) and 2 (Different Angles $) \times 2$
(Instruction Set), for each experiment. We also used two different experimental designs, one with instruction set between participants involving a study block followed by a test block (Exps. 1a, 1b, 3a, and 3b, similar to the design of Gagnier \& Intraub, 2012), and a within-participants design with multiple pictures in a trial, testing one immediately (Exps. 2a and 2b), in which participants were given imagery instructions in a second block (similar to the design of Dickinson \& Intraub, 2008).

The use of multiple methodologies allowed us to assess any effects of instructions on $\mathrm{BE}$ in a range of contexts. We expected to find (a) significant $\mathrm{BE}$ in both $\mathrm{CC}$ and $\mathrm{WW}$ conditions, (b) significantly greater BE in WC than in $\mathrm{CW}$ conditions, and (c) significantly greater BE with elaboration instructions than with standard instructions. Finally, in the experiments that included imagery measures, we expected to see a positive correlation between imagery and BE. 


\section{Experiments 1a and 1b}

Photographs of scenes (both close-up and wide-angle views) were presented, and participants were either given standard instructions or prompted to imagine sounds and odors that would accompany each scene. At test, the photographs were either the same as (CC, WW trials) or different from (CW, WC trials) the ones given at study, and participants rated whether the test photograph was closer, the same, or farther away, on a 5-point scale (Intraub \& Richardson, 1989). Importantly, rating an identical photograph as closer would indicate BE for the original view. Of interest was whether imagining additional details would increase BE, as participants confused imagined with experienced details. In Experiment 1a, participants studied each photograph for $6 \mathrm{~s}$ during the first block, and Experiment $1 \mathrm{~b}$ photographs were presented for $15 \mathrm{~s}$ of study.

In addition to manipulating the instructional sets (standard vs. elaboration), Experiment $1 \mathrm{~b}$ also included the Vividness of Visual Imagery Questionnaire (VVIQ; Marks, 1973), which assesses pictorial imagery. Marks had participants study photographs and, following a distraction task, asked multiplechoice questions to assess picture memory (e.g., "Was the ballerina standing: on tiptoes, with one foot on the floor, or with both feet on the floor?," p. 19). He found more accurate picture memory for participants with higher VVIQ scores (self-reported experiencing of more vivid images). A parallel result would be a negative correlation between VVIQ and BE, with higher VVIQ scores corresponding to more accurate memory (less or no BE); however, more recent work has suggested that higher VVIQ scores instead correspond with increased BE (Markham \& Hynes, 1993; Mullally et al. 2012).

\section{Method}

\section{Participants}

Undergraduates participated in either Experiment 1a $(n=67$; 40 women, $27 \mathrm{men})$ or $1 \mathrm{~b}(n=58 ; 37$ women, $21 \mathrm{men})$ as one option to earn course credit.

\section{Materials}

The stimuli were 48 photographs: 12 each of city scenes, outdoor market scenes, desert scenes, and forested scenes from various locations around the world. There were two versions of each picture: a wide-angle view, as well as a close-up that was either $10 \%, 15 \%$, or $20 \%$ enlarged and then cropped to the same size as the original $(720 \times 480$ pixels $)$. Dickinson and Intraub (2008) had close-up views that were $8 \%-21 \%$ enlargements of the wide-angle views. Both the wide-angle and close-up versions were the same size, but more background was visible in the wide-angle versions (see Fig. 1 for examples). All of the pictures were in landscape orientation and were presented centered on a black screen.

Experiment 1b included the VVIQ (Marks, 1973), which assesses pictorial imagery. Each of four scenes (a friend or relative, the rising sun, the front of a shop, and a country scene) was to be imagined once with the participant's eyes open and once with the eyes closed. Participants rated the vividness of each mental image on a 5 -point scale $(1=$ Perfectly clear and as vivid as normal vision, $5=$ No image at all, you only "know" that you are thinking of an object; Marks, 1973, p. 24); note that lower scores indicate superior visual imagery.

\section{Apparatus}

Experiment 1a was run on a Dell Optiplex 9020 computer (1, $280 \times 1024$ screen resolution). In Experiment $1 \mathrm{~b}$ we used a 21 -in. iMac display $(1,920 \times 1,080$ screen resolution). In both experiments, the stimuli were presented by and responses were collected using SuperLab (Cedrus).

\section{Design}

Experiments 1a and $1 \mathrm{~b}$ were based on a 4 (Trial Type: CC, $\mathrm{WW}, \mathrm{CW}, \mathrm{WC}) \times 2$ (Condition: standard, elaboration) mixedfactor design with Condition as the between-participants factor.

\section{Procedure}

Participants viewed each study photograph for either $6 \mathrm{~s}$ (Exp. 1a) or $15 \mathrm{~s}$ (Exp. 1b). The opening instructions asked participants to try to remember as much detail as possible, including the background of the photograph. In the standard condition for both Experiments 1a and $1 \mathrm{~b}$, participants initially viewed a photograph without additional instructions. Text then appeared above the photograph to indicate that the participants had $4 \mathrm{~s}$ (Exp. 1a) or $10 \mathrm{~s}$ (Exp. 1b) left to study it, followed by text appearing below the photograph to indicate that participants had $2 \mathrm{~s}$ (Exp. 1a) or $5 \mathrm{~s}$ (Exp. 1b) left to study.

Participants in the experimental conditions in both Experiments $1 \mathrm{a}$ and $1 \mathrm{~b}$ received additional elaboration instructions to imagine sounds and odors that might be part of the scene, suggesting that this might help their memory. Instead of text indicating the remaining time, text appeared above each photograph asking participants to imagine sounds after $2 \mathrm{~s}$ (Exp. 1a) or $5 \mathrm{~s}$ (Exp. 1b), followed by text appearing below the photograph asking participants to imagine odors when $2 \mathrm{~s}$ (Exp. 1a) or $5 \mathrm{~s}$ (Exp. 1b) of study remained. The photographs were counterbalanced across participants, such that each version (close-up or wide-angle) appeared as a study picture. 
For both experiments, immediately after the study phase, the test phase began. Instructions indicated that the next photographs would be the same or would be similar to the ones just shown, and that the task was to decide whether each photograph was taken from the same camera position as the version seen earlier, or whether the camera was a little (or a lot) closer or farther away. Participants were then shown an example study photograph with test photographs that were labeled a lot closer, a little closer, a little farther away, and a lot farther away. After any questions were addressed, participants saw test photographs and categorized each one using a 5-point scale [a lot closer (-2), a little closer (-1), the same $(0)$, a little farther away $(+1)$, and a lot farther away (+2)] to indicate any changes relative to the version they had seen at study, using virtual onscreen buttons for mouse responses. Participants also indicated their confidence as being sure, pretty sure, not sure, or did not see the picture. In Experiment 1b, participants were then escorted to a different room, and the VVIQ was administered by a research assistant who read the questions and recorded participants' verbal responses. The VVIQ was always administered after the BE task, to avoid suggesting that participants should use imagery in the standard-instruction condition.

\section{Results}

\section{Confidence ratings}

Participants were confident in their ratings: for the $6 \mathrm{~s}$ (Exp. 1a) elaboration instructions, they reported being sure, pretty sure, and not sure $28 \%, 51 \%$, and $17 \%$, respectively; for the standard instructions, these responses were $32 \%, 47 \%$, and $18 \%$, respectively. Participants

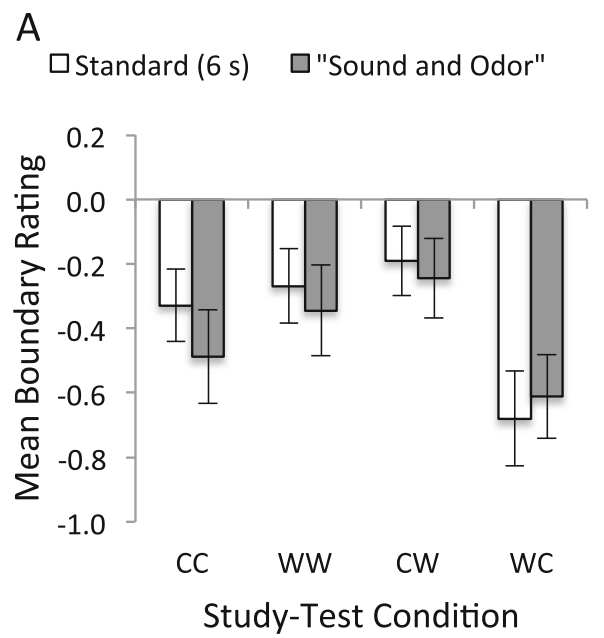

Fig. 2 Mean boundary ratings for the standard and elaboration conditions in Experiments 1a (A) and 1b (B). The experimental group was asked to imagine sounds one might hear and odors one might smell for a scene. (A) Study time at $6 \mathrm{~s}$ for the standard and elaboration conditions. (B) Study time at $15 \mathrm{~s}$ for the standard and elaboration indicated that they did not remember the picture $4 \%$ of the time under both the elaboration and standard instructions. For the 15-s condition (Exp. 1b), the elaboration instruction participants were sure, pretty sure, and not sure $34 \%, 46 \%$, and $17 \%$ of the time; for the standard instruction participants, theses values were $34 \%, 45 \%$, and $16 \%$, respectively. The participants in Experiment $1 \mathrm{~b}$ chose did not see picture $2 \%$ with elaboration, and $6 \%$ with standard, instructions.

\section{BE scores}

The mean boundary ratings and $95 \%$ confidence intervals are presented in Fig. 2. For both experiments, in the first of the three critical comparisons, significant negative ratings for both the CC and WW trials indicate BE for both types of "same" trials. Second, participants gave larger ratings to WC than to $\mathrm{CW}$ trials. Third, there was no effect of instruction condition ("sound and odor" vs. standard) in either experiment.

Experiment 1a (6-s study phase) For the "same" trials, a 2 (Trial Type: CC, WW) $\times 2$ (Instruction Set: standard, elaboration) mixed-design ANOVA showed a marginal main effect of trial type, $F(1,65)=3.46, p=.07, \eta^{2}=.05$, indicating similar BE for $\mathrm{CC}$ and $\mathrm{WW}$ trials, with a trend toward more $\mathrm{BE}$ in $\mathrm{CC}$ than in WW. We observed no main effect of instructions, $F(1,65)=2.39, p=.13, \eta^{2}=.04$, nor an interaction, $F(1$, $65)<1$. For "different" trials, a 2 (Trial Type: CW, WC) $\times 2$ (Instruction Set: standard, elaboration) mixed-design ANOVA revealed a main effect of trial type, $F(1,65)=69.11, p<.001$, $\eta^{2}=.51$, indicating that participants gave larger ratings to $\mathrm{WC}$ changes from study to test than they gave to $\mathrm{CW}$ changes, replicating previous work (e.g., Dickinson \& Intraub, 2008).

\section{B}

$\square$ Standard (15 s) $\square$ "Sound and Odor"

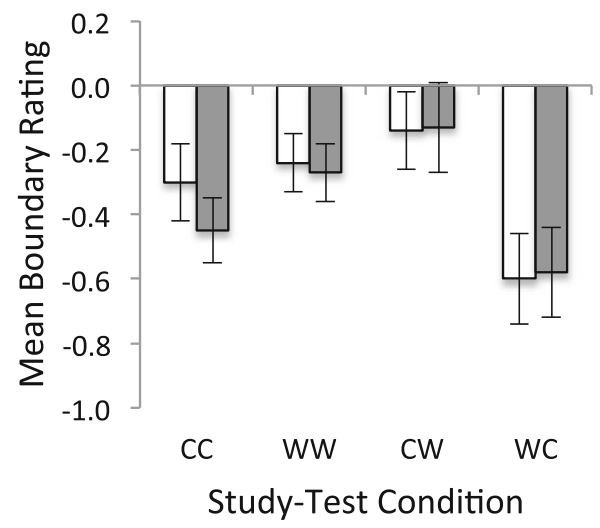

conditions. Negative ratings indicate that the test scene appeared to be closer than the original, and ratings of 0 indicate that the test scene appeared identical to the original. Error bars indicate $95 \%$ confidence intervals of the means. $\mathrm{C}=$ close-up, $\mathrm{W}=$ wide-angle 
No main effect of instructions emerged, $F(1,65)<1$, nor an interaction, $F(1,65)=1.40, p=.24, \eta^{2}=.01$.

Experiment 1b (15-s study phase) For the "same" trials, a 2 (Trial Type: CC, WW) $\times 2$ (Instruction Set: standard, elaboration) mixed-design ANOVA revealed a main effect for trial type, $F(1,56)=8.78, p=.004, \eta^{2}=.13$, indicating stronger $\mathrm{BE}$ for $\mathrm{CC}$ than for WW trials. No main effect of instructions was apparent, $F(1,56)=1.73, p=.19, \eta^{2}=.03$, nor an interaction, $F(1,56)=2.55, p=.12, \eta^{2}=.04$. For "different" trials, a 2 (Trial Type: $\mathrm{CW}, \mathrm{WC}) \times 2$ (Instruction Set: standard, elaboration) mixed-design ANOVA revealed a main effect of trial type, $F(1,56)=70.99, p<.001, \eta^{2}=.56$, indicating that participants gave larger ratings to WC changes from study to test than they gave to $\mathrm{CW}$ changes, replicating previous work (e.g., Dickinson \& Intraub, 2008). There was no main effect of instructions, $F(1,56)<1$, nor an interaction, $F(1,56)<1$.

\section{Pictorial imagery}

We found individual differences in self-reported imagery ability in Experiment $1 \mathrm{~b}$ : There was a significant difference between the top $(M=1.67, S D=0.24)$ and bottom $(M=3.13$, $S D=0.43)$ quartiles for the VVIQ scores, $t(26)=11.12, p<$ $.001, d=4.20$. However, we found no difference between the $\mathrm{CC}$ scores for those in the top $(M=-0.42, S D=0.39)$ and bottom $(M=-0.21, S D=0.27)$ quartiles of the VVIQ scores, $t(26)=1.68, p=.10, d=0.63$. In other words, participants who self-reported having strong visual imagery had only marginally greater (more negative) BE than those who selfreported poor pictorial imagery. Further emphasizing the lack of a link between pictorial imagery ability and BE, we observed no significant correlation between VVIQ scores and the $\mathrm{CC}$ test condition (since $\mathrm{CC}$ is thought to be the most sensitive test of BE; see Intraub, Daniels, Horowitz, \& Wolfe, 2008), $r(57)=.16$, n.s.

\section{Discussion}

BE was observed in both angle sizes of "same" trials (CC and WW), with the typical pattern of more BE for CC trials being observed with longer presentation times at study (Exp. 1b, $15 \mathrm{~s}$ ). In addition, participants gave larger ratings to WC trials than to $\mathrm{CW}$ trials, though they rated even $\mathrm{CW}$ trials as being closer, suggesting that this picture set led to strong BE. We did not find a significant relationship between self-reported visual imagery and BE scores.

Participants instructed to imagine additional informationspecifically, sounds and odors-did show significant BE across "same" and "different" trials. However, the BE ratings following elaboration instructions were equivalent to those after standard instructions. Unlike memory for actions (Thomas et al. 2003), there was not an inflation of memory errors following more elaborate encoding for BE. We also found no reduction of $\mathrm{BE}$, as had been observed for imagined objects (Foley et al. 2010), suggesting that memory was not improved by the act of imagining additional details.

One possible objection to Experiments $1 \mathrm{a}$ and $1 \mathrm{~b}$ is that the photographs used in them were not typical of BE studies, which feature pictures with a single object on a continuous background. We originally chose the more complicated, multi-object scenes in order to facilitate imagining additional information, but it is important to examine whether an imagination inflation effect would be possible with simpler scenes. Perhaps in the same way that simpler line drawings lead to greater BE than do photographs (Gagnier \& Intraub, 2012), simpler photographs might allow for more of an imagination effect.

\section{Experiments 2a and 2b}

As was noted in the introduction, our goal was to examine as thoroughly as possible whether explicit imagination can influence $\mathrm{BE}$ by using both types of BE methods across our experiments. Thus, Experiments $2 \mathrm{a}$ and $2 \mathrm{~b}$ also involved a different method, in which a series of pictures was presented followed by an immediate memory test using one of the pictures, similar to the design of Dickinson and Intraub (2008). The first block presented standard instructions, and a second block presented elaboration instructions to imagine sounds and odors; this within-participants design also increased the range of conditions under which we explored the relationship between imagination and BE.

Furthermore, in Experiment $2 \mathrm{~b}$ we used a new set of photographs, each featuring a single central object (see Fig. 3). The instructions asked participants to imagine how traffic sounds, food tastes, and animals smelled.

Finally, due to mixed reports regarding satisfactory testretest reliability for the VVIQ (McAvinue \& Robertson, 2007), we adopted a new measure of visual imagery: All participants completed the session by taking the Object-Spatial Imagery Questionnaire (OSIQ; Blajenkova, Kozhevnikov, \& Motes, 2006), which consists of questions that reliably distinguish object imagery (representations of color and individual objects) from spatial imagery (representations of schematic images and spatial relations between objects).

\section{Method}

\section{Participants}

Undergraduates participated in either Experiment $2 \mathrm{a}(n=15$; 9 women, 6 men) or $2 \mathrm{~b}$ ( $n=31 ; 18$ women, 13 men), both of which had within-participants designs, as one option to earn course credit. 
Fig. 3 Schematic of a trial sequence during the imagine block for Experiment 2b, using photographs from our typical set. The actual stimuli did not fill the entire screen, and all images and instructions were centered on a black background

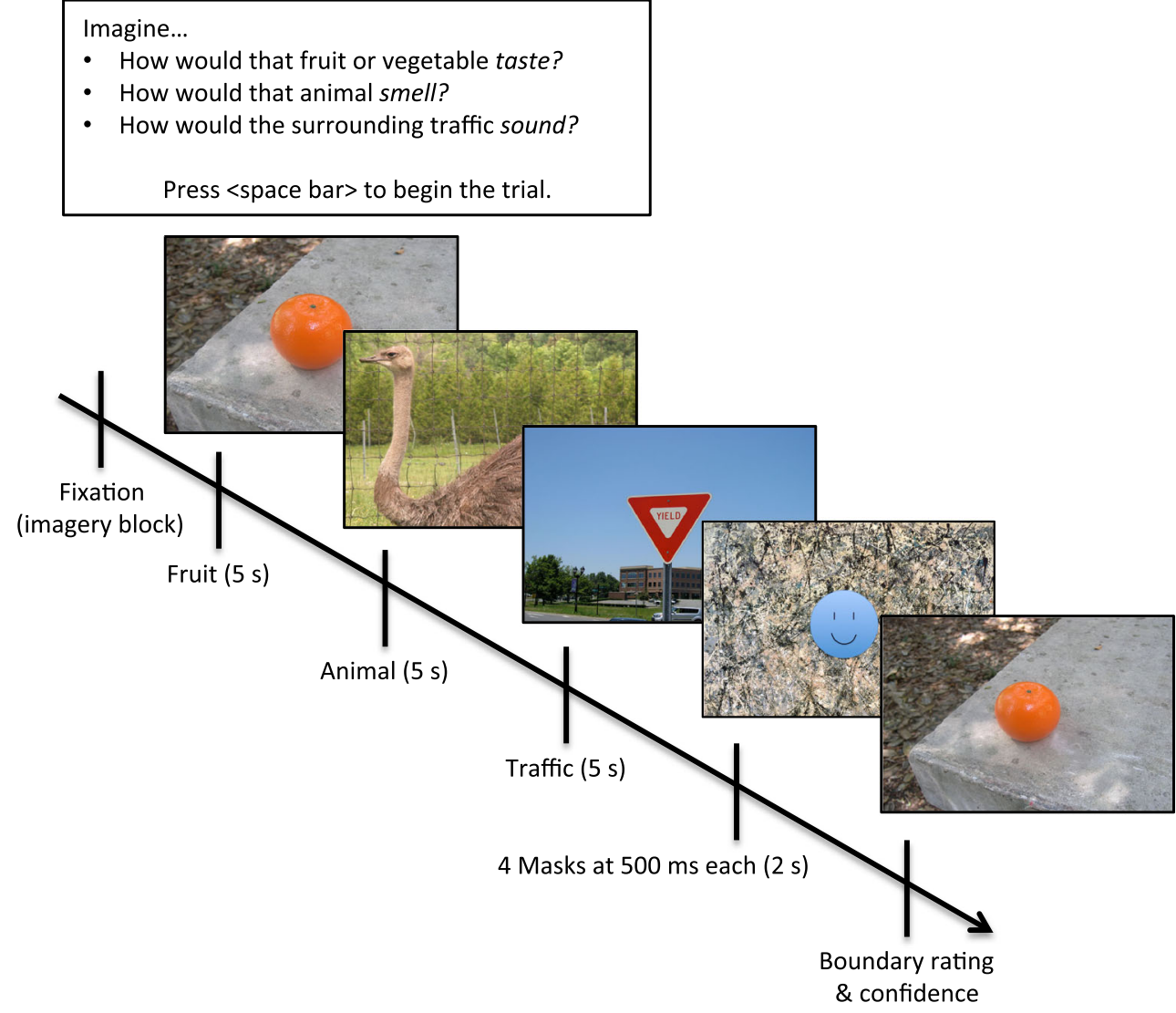

\section{Materials}

Experiment 2a was based on the same photographs as Experiment 1. New photographs were taken for Experiment $2 \mathrm{~b}$, each featuring a single, central object featuring a single piece of fruit, animal, or traffic sign (eight in each category; see Fig. 3). There were two versions of each picture: a wide-angle view, and a close-up that was either $10 \%, 15 \%$, or $20 \%$ enlarged and then cropped to the same size as the original (720 $\times 480$ pixels). As we noted earlier, Dickinson and Intraub (2008) had close-up views that enlarged wideangle views from $8 \%$ to $21 \%$. Both the wide-angle and close-up versions were the same size, but more background was visible in the wide-angle versions. All of the pictures were in landscape orientation and were presented centered on a black screen.

Both Experiments $2 \mathrm{a}$ and $2 \mathrm{~b}$ included the OSIQ (Blajenkova et al. 2006), which consists of 30 intermixed questions, 15 measuring object imagery (representations of color and individual objects) and 15 measuring spatial imagery (representations of schematic images and spatial relations between objects). Participants responded on a 5-point scale (1 $=$ totally disagree, $5=$ totally agree $)$, note that higher scores indicate superior visual imagery ability.
Design

Experiments $2 \mathrm{a}$ and $2 \mathrm{~b}$ featured 4 (Trial Type: CC, WW, CW, WC) $\times 2$ (Instructions: standard, elaboration) withinparticipants designs.

\section{Procedure}

Participants were tested individually with the same equipment as in Experiment $1 \mathrm{~b}$. Unlike the blocked procedure used in Experiments $1 \mathrm{a}$ and $1 \mathrm{~b}$, Experiments $2 \mathrm{a}$ and $2 \mathrm{~b}$ used a rapid serial visual presentation (RSVP) procedure (see Fig. 3 for an Experiment $2 \mathrm{~b}$ RSVP series of events). Initial instructions explained that participants would see a sequence of pictures and highlighted the importance of remembering the entire picture, including the background. The instructions included an example study photograph with test photographs that were labeled a lot closer, a little closer, a little farther away, and a lot farther away. Each trial consisted of an RSVP sequence (Exp. 2a: $4 \mathrm{~s} /$ photograph, four photographs per RSVP; Exp. $2 \mathrm{~b}: 5 \mathrm{~s} /$ photograph, three photographs per RSVP). Following the last picture, four smiley faces were presented in sequence $(500 \mathrm{~ms} / \mathrm{smiley}$, total of $2,000 \mathrm{~ms})$ as a mask, and then one of the study pictures was tested (see Fig. 3). The smiley faces were centered on a random mask and filled approximately $8 \%$ 
of the total area (see Fig. 3). Participants rated position and confidence as in Experiments 1a and 1b. Each RSVP sequence was repeated (Exp. 2a, eight times; Exp. 2b, six times), so that each picture was included in a "same" trial (CC or WW) and a "different" trial (CW or WC). Experiment $2 \mathrm{a}$ included 96 trials, and Experiment $2 \mathrm{~b}$ included 48 trials. Nothing about the RSVP sequence indicated which picture would be tested, and there were equal numbers of the study-test conditions ( $\mathrm{CC}, \mathrm{WW}, \mathrm{CW}, \mathrm{WC}$ ) across the sequences.

All participants began with standard BE instructions. After the first block (Exp. 2a, 48 trials; Exp. 2b, 24 trials), new instructions appeared explaining that to help with the memory task, participants should now try to imagine additional details. The Experiment $2 \mathrm{a}$ imagery instructions prompted participants to imagine sounds and odors (like those in Exps. 1a and 1b). Experiment $2 \mathrm{~b}$ asked how the fruit would taste, how the animal would smell, and how the surrounding traffic would sound. These elaboration instructions were repeated before each trial. A different set of pictures was used in each block, and between participants the sets were counterbalanced so that both sets were used in the standard block and the elaboration block. Trials were presented in a unique random order for each participant.

Following the BE trials, the final block of the experiment was the OSIQ, with participants indicating their agreement to each statement using the keypad. The order of the blocks was fixed to avoid suggesting that participants use of imagery during the standardinstruction condition for the BE task.

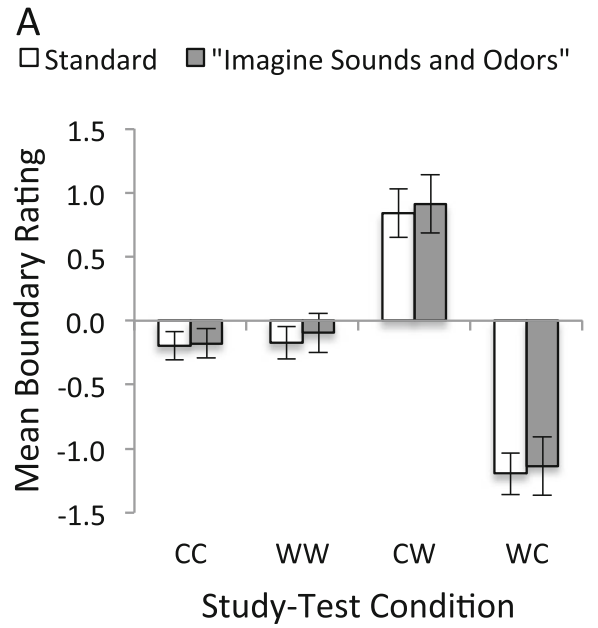

Fig. 4 Mean boundary ratings for the standard and elaboration conditions in Experiments 2a (A) and 2b (B). All participants began with standard conditions, and in a second block were asked to (A) imagine sounds and odors for the world-picture set, or (B) imagine taste, odor, and sounds for the typical-picture set. Negative ratings

\section{Results}

\section{Confidence ratings}

Participants were confident in their ratings: For the world set of pictures (Exp. 2a: $4 \mathrm{~s} /$ photograph, four photographs per RSVP) with elaboration instructions, participants reported being sure, pretty sure, and not sure $43 \%, 44 \%$, and $13 \%$, respectively; for the standard-instruction, these responses were $43 \%, 42 \%$, and $14 \%$, respectively. Participants indicated that they did not remember the picture on $1 \%$ of trials under elaboration instructions, and they never did not remember under standard instructions. For the typical set of pictures (Exp. 2b: 5 s/photograph, three photographs per RSVP) with elaboration instructions, participants were also confident, with sure, pretty sure, and not sure responses on $49 \%, 41 \%$, and $9 \%$ of trials; for the standard instructions, these values were $52 \%, 40 \%$, and $8 \%$, respectively. Participants chose did not see picture on less than $1 \%$ of trials in both the elaboration and standard conditions.

\section{BE scores}

The mean boundary ratings and $95 \%$ confidence intervals are presented in Fig. 4. For both experiments, in the first of the three critical comparisons, significant negative ratings for both CC and WW trials indicated BE for both types of "same" trials. Second, participants gave larger ratings to $\mathrm{WC}$ than to $\mathrm{CW}$ trials. Third, we found no effect of instruction condition (Exp. 2a, "sound and odor"; Exp. 2b, "taste, odor, and sound," compared with standard) in either experiment.

\section{B}

$\square$ Standard $\square$ "Imagine Taste, Odor, and Sounds"

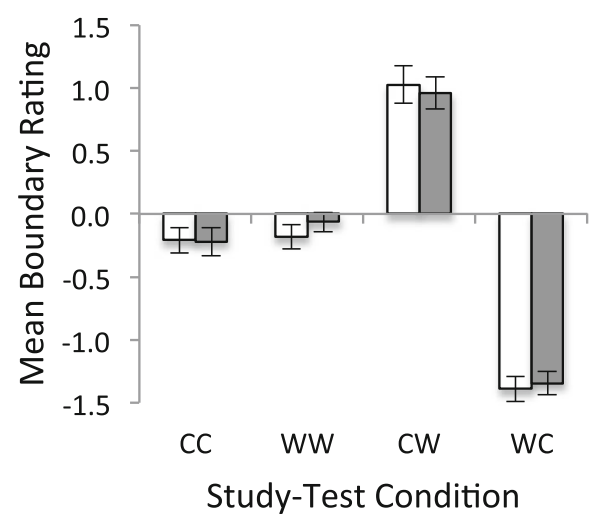

indicate that the test scene appeared to be closer than the original, and ratings of 0 indicate that the test scene appeared identical to the original. Error bars indicate $95 \%$ confidence intervals of the means. C $=$ close-up, $\mathrm{W}=$ wide-angle 
Experiment 2a (world pictures, 4 s/photograph, four photographs per RSVP) For the "same" trials, a 2 (Trial Type: $\mathrm{CC}, \mathrm{WW}) \times 2$ (Instruction Set: standard, elaboration) withinparticipants ANOVA revealed no main effect of trial type, $F(1$, $14)<1$, indicating equivalent $\mathrm{BE}$ for $\mathrm{CC}$ and $\mathrm{WW}$ trials; no main effect of instructions, $F(1,14)<1$; and no interaction, $F(1,14)<1$. For "different" trials, a 2 (Trial Type: CW, WC) $\times$ 2 (Instruction Set: standard, elaboration) within-participants ANOVA revealed a main effect of trial type, $F(1,14)=$ $11.36, p<.01, \eta^{2}=.21$, indicating that participants gave larger ratings to $\mathrm{WC}$ changes than to $\mathrm{CW}$ changes from study to test, replicating previous work (e.g., Dickinson \& Intraub, 2008); no main effect of instructions, $F(1,14)<1$; and no interaction, $F(1,14)=1.64, p=.22, \eta^{2}=.01$. Note that the absolute values of the WC scores were used in the ANOVA, in order to compare participants' use of the scale (Dickinson \& Intraub, 2008)

Experiment 2b (typical pictures, 5 s/photograph, three photographs per RSVP) For the "same" trials, a 2 (Trial Type: CC, WW) $\times 2$ (Instruction Set: standard, elaboration) within-participants ANOVA revealed no main effect of trial type, $F(1,30)=2.71, p=.11, \eta^{2}=.04$, indicating equivalent $\mathrm{BE}$ for $\mathrm{CC}$ and WW trials. We observed no main effect of instructions, $F(1,30)=2.09, p=.16, \eta^{2}=.01$, and no interaction, $F(1,30)=2.22, p=.15, \eta^{2}=.02$. For "different" trials, a 2 (Trial Type: CW, WC) $\times 2$ (Instruction Set: standard, elaboration) within-participants ANOVA revealed a main effect of trial type, $F(1,30)=50.30, p<.001, \eta^{2}=.39$, indicating that participants gave larger ratings to $\mathrm{WC}$ changes than to CW from study to test, replicating previous work (e.g., Dickinson \& Intraub, 2008). No main effect of instructions was apparent, $F(1,30)=1.90, p=.18, \eta^{2}=.00$, and no interaction, $F(1,30)<1$. Note that the absolute values of the WC scores were used in the ANOVA, in order to compare participants' use of the scale (Dickinson \& Intraub, 2008).

\section{Object and spatial imagery}

We found individual differences in self-reported imagery ability in both experiments, with a significant difference between the top and bottom quartiles for the OSIQ object imagery and spatial imagery scores (see Table 1). In Experiment 2a (world pictures, $4 \mathrm{~s} /$ photograph, four photographs per RSVP), the top and bottom quartiles for object imagery were statistically different, $t(6)=5.95, p<.01, d=4.21$; the same pattern held for spatial imagery, $t(6)=15.39, p<.001, d=10.89$. Experiment 2b (typical pictures, $5 \mathrm{~s} /$ photograph, three photographs per RSVP) replicated that pattern: $t(14)=10.02, p<.001, d=$ 5.01 , and $t(14)=11.91, p<.001, d=5.95$, for object and spatial imagery, respectively. However, we observed no difference between the $\mathrm{CC}$ scores for those in the top and bottom quartiles of the OSIQ subscale in either Experiment $2 \mathrm{a}$ or $2 \mathrm{~b}$,
Table 1 Mean (with $S D$ ) scores on the Object-Spatial Imagery Questionnaire (OSIQ) for the top and bottom quartiles, calculated separately for the object and spatial imagery measures

OSIQ Measure

\begin{tabular}{lcc}
\cline { 2 - 3 } Quartile & \multicolumn{1}{c}{ Object Imagery } & Spatial Image \\
\hline Experiment 2a (World Pictures) & \\
Top & $\mathbf{3 . 8 3}(0.18)$ & $\mathbf{3 . 4 2}(0.15)$ \\
Bottom & $\mathbf{2 . 4 5}(0.43)$ & $\mathbf{2 . 1 0}(0.09)$ \\
Experiment $2 b$ (Typical Pictures) & \\
Top & $\mathbf{3 . 9 9}(0.28)$ & $\mathbf{3 . 5 2}(0.26)$ \\
Bottom & $\mathbf{2 . 5 2}(0.30)$ & $\mathbf{1 . 9 7}(0.26)$ \\
Experiment 3a (World Pictures) & \\
Top & $\mathbf{4 . 0 4}(0.18)$ & $\mathbf{3 . 4 5}(0.27)$ \\
Bottom & $\mathbf{2 . 8 7}(0.26)$ & $\mathbf{2 . 2 2}(0.24)$
\end{tabular}

Quartiles are calculated separately for object and spatial imagery $(1=$ totally disagree, 5 = totally agree), with higher scores representing higher self-reported imagery. Bold means indicate that the top and bottom quartiles were significantly different (see the text for the statistical details of each comparison).

all $t \mathrm{~s}<1.55$, n.s. (see Table 2 for the BE scores). In other words, participants who self-reported having strong object imagery or spatial imagery had BE equivalent to those who self-reported poor object imagery or spatial imagery. Further emphasizing the lack of a link between self-reported visual imagery ability and BE, there were no significant correlations ( $r$ s between -.46 and .36, n.s.) between OSIQ scores and any of the $\mathrm{CC}$ conditions (since $\mathrm{CC}$ is thought to be the most sensitive test of BE; see Intraub et al. 2008).

\section{Discussion}

BE was observed in all $\mathrm{CC}$ trials, standard and elaboration, and for WW trials in the standard condition. When participants rated WW trials following elaboration instructions, no BE was observed (see Fig. 4). Participants again gave larger ratings to $\mathrm{WC}$ than to $\mathrm{CW}$ trials, and using the RSVP method of testing BE, they correctly identified that CW trials contained wider images (positive scores). We found no relationship between self-reported visual imagery and BE scores.

Two interesting patterns have emerged from Experiments 2a (world pictures, $4 \mathrm{~s} /$ photograph, four photographs per RSVP stream) and 2b (typical pictures, $5 \mathrm{~s} /$ photograph, three photographs per RSVP stream) concerning stimuli and method. First, visual inspection of Fig. 4 reveals practically identical patterns for the BE ratings of the world set of photographs and the more typical, single-item set of photographs. This strengthens our confidence that the data patterns reported in Experiments $1 \mathrm{a}, 1 \mathrm{~b}$, and $2 \mathrm{a}$ are not unique to relatively complicated scenes. Second, participants correctly noticed that the $\mathrm{CW}$ pictures were actually wider (see the positive ratings in 
Table 2 Mean (with $S D$ ) boundary extension (BE) scores for the top and bottom quartiles of Object-Spatial Imagery Questionnaire (OSIQ) measures

\begin{tabular}{|c|c|c|c|c|}
\hline \multirow[b]{3}{*}{ Quartile } & \multicolumn{4}{|l|}{ OSIQ Factor } \\
\hline & \multicolumn{2}{|l|}{ Object Imagery } & \multicolumn{2}{|c|}{ Spatial Imagery } \\
\hline & CC Standard & CC Elaboration & CC Standard & CC Elaboration \\
\hline \multicolumn{5}{|c|}{ Experiment 2a (World Pictures) } \\
\hline Top & $-0.33(0.22)$ & $-0.10(0.11)$ & $-0.23(0.22)$ & $-0.10(0.33)$ \\
\hline Bottom & $-0.10(0.21)$ & $-0.25(0.26)$ & $-0.27(0.25)$ & $-0.33(0.22)$ \\
\hline \multicolumn{5}{|c|}{ Experiment $2 \mathrm{~b}$ (Typical Pictures) } \\
\hline Top & $-0.12(0.20)$ & $-0.14(0.32)$ & $-0.33(0.31)$ & $-0.27(0.40)$ \\
\hline Bottom & $-0.25(0.43)$ & $-0.44(0.43)$ & $-0.12(0.21)$ & $-0.27(0.34)$ \\
\hline
\end{tabular}

BE scores were sorted by each OSIQ factor; negative scores indicates BE. No differences in BE scores were observed based on OSIQ factors. CC, close-up stimuli at both study and test
Fig. 4) when the RSVP method was used. However, participants still gave larger ratings to the WC condition, shown in the more extreme ratings than in the $\mathrm{CW}$ condition, replicating previous work (e.g., Dickinson \& Intraub, 2008).

\section{Experiments 3a and 3b}

After failing to find an effect on BE from cross-modal elaboration (e.g., "imagine the sounds and smells related to what is presented visually"), we attempted to detect it using visual elaboration. Specifically, we asked participants to imagine what might be to the left or right of a scene (Exp. 3a) or to zoom in or out of a picture, much as a photographer can (Exp. $3 \mathrm{~b}$; see Fig. 5 for examples), to see whether visual elaboration affects BE. Intraub (2012) discusses the modality of the input (vision vs. haptics) being the key to the amount of boundary extension, with larger BE being supported by visual exploration of a scene. The present experiments returned to the methods of Experiment 1b, but now we instructed participants to imagine additional visual information.

\section{Method}

\section{Participants}

Undergraduates participated in either Experiment $3 \mathrm{a}(n=70$; 39 women, 31 men) or $3 \mathrm{~b}(n=49 ; 35$ women, 14 men $)$ as one option to earn course credit.

\section{Materials}

The same picture stimuli from Experiments $1 \mathrm{a}, 1 \mathrm{~b}$, and 2a were used in Experiments $3 \mathrm{a}$ and $3 \mathrm{~b}$. Experiment $3 \mathrm{a}$ included the OSIQ (Blajenkova et al. 2006). Participants responded on a 5 -point scale $(1=$ totally disagree, $5=$ totally agree $)$; note that higher scores indicate superior visual imagery ability.

\section{Design}

Experiment 3a was based on a 4 (Trial Type: CC, WW, CW, WC) $\times 2$ (Condition: standard, elaboration) mixed-factor design with Condition as the between-participants factor. Experiment 3b had a 4 (Trial Type: CC, WW, CW, WC) $\times 3$ (Condition: standard, elaboration zoom in, elaboration zoom out) mixed-factor design with Condition as the betweenparticipants factor.

\section{Procedure}

The procedure was identical to that of Experiment $1 \mathrm{~b}$ (15 s of study exposure), but now run on the same apparatus as Experiment 1a and with "imagine left-right" and "zoom" instructions. In Experiment 3a, participants in the elaboration condition viewed the picture without instructions for $5 \mathrm{~s}$. Text then appeared above the photograph to instruct participants to imagine what the photographer would see to the left of the scene $(5 \mathrm{~s})$. Finally, text appeared below the photograph to instruct participants to imagine what the photographer would see to the right of the scene $(5 \mathrm{~s})$. Following the BE trials, the final block of the experiment was the OSIQ, with participants indicating their agreement to each statement using the keypad.

In Experiment $3 b$, the participants in the elaboration conditions were shown two examples of what it would look like if a photographer zoomed in $80 \%$ and $60 \%$ (or out $120 \%$ and $140 \%$; see Fig. 5 for the examples used in the instructions). Participants viewed each picture without instructions for $5 \mathrm{~s}$. Text then appeared above the photograph to instruct participants to imagine what they would see if they zoomed in $80 \%$ (or out $120 \%$ ) for $5 \mathrm{~s}$. Finally, text appeared below the photograph to instruct participants to imagine what they would see if they zoomed in $60 \%$ (or out $140 \%$ ). The OSIQ was not included in Experiment 3b, due to the experimental session duration. 
Fig. 5 Examples presented to participants in Experiment 3b: Zooming in (A) and zooming out (B)

\section{A. Zooming in, you would see less of the horizon... Original view $80 \%$}
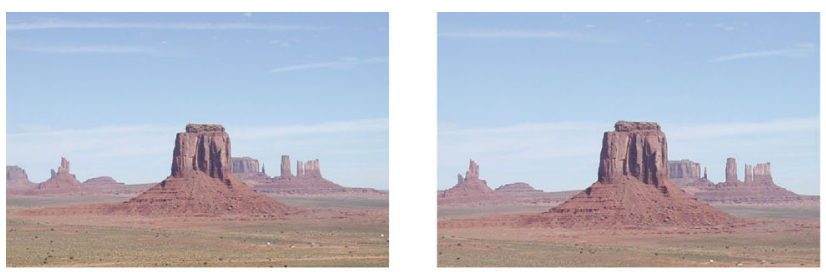

\section{$60 \%$}

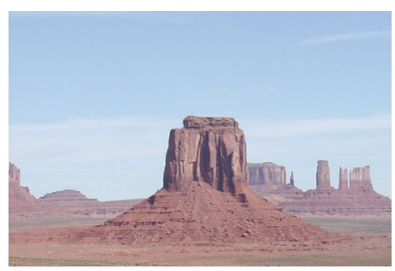

Zooming in, you would see less of the building... Original view $80 \%$
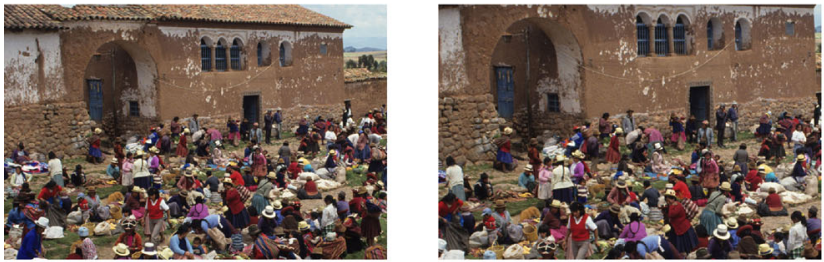

B. Zooming out, you would see more of the horizon...

\section{Original view}

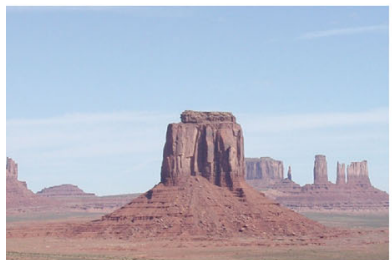

Zooming out, you would see more of the building... Original view

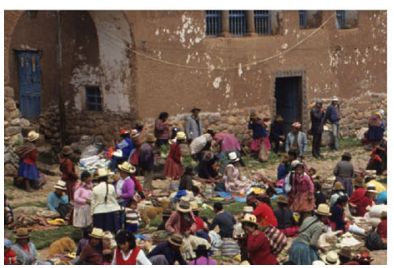

$120 \%$

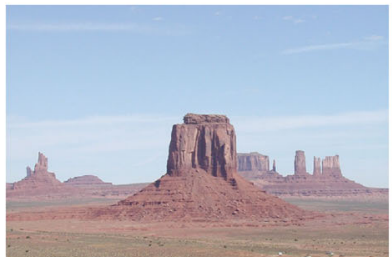
$120 \%$

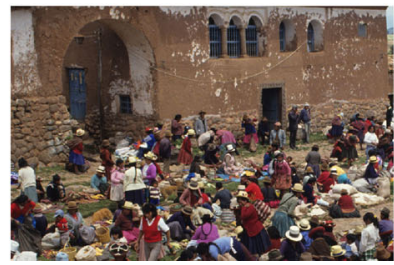

\section{$140 \%$}

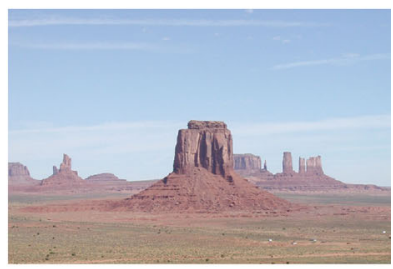

$140 \%$

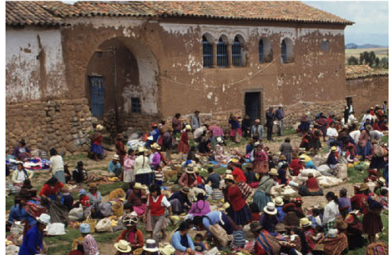

\section{Results}

\section{Confidence ratings}

Participants were confident in their ratings: for the left-right (Exp. 3a) elaboration instructions, they reported being sure, pretty sure, and not sure on $41 \%, 41 \%$, and $14 \%$ of trials, respectively; for the standard instructions, these responses were $39 \%, 40 \%$, and $17 \%$ of the total. Participants indicated that they did not remember the picture on $3 \%$ of trials under elaboration instructions, and on $4 \%$ under standard instructions. For the zoom conditions (Exp. 3b), with zoom-in elaboration instructions participants were also confident-33\%, $50 \%$, and $13 \%$; for zoom-out elaboration, these values were

$31 \%, 46 \%$, and $17 \%$; and for the standard condition, they were $30 \%, 56 \%$, and $14 \%$. Participants chose did not see picture on $3 \%$ of trials for zoom in, on $6 \%$ for zoom out, and on $1 \%$ for standard.

\section{BE scores}

The mean boundary ratings and $95 \%$ confidence intervals are presented in Fig. 6. For both experiments, in the first of the three critical comparisons, significant negative ratings for both $\mathrm{CC}$ and WW trials indicated BE for both types of "same" trials. Second, participants gave larger ratings to $\mathrm{WC}$ than to $\mathrm{CW}$ trials. Third, we found no effect of instruction condition 
A

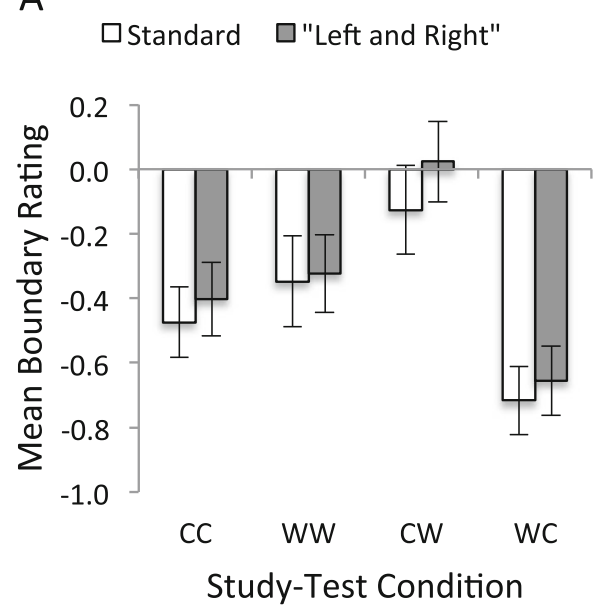

Fig. 6 Mean boundary ratings for the standard and elaboration conditions in Experiments $3 \mathrm{a}$ (A) and $3 \mathrm{~b}$ (B). (A) The experimental group was asked to imagine what was to the left and right of each scene at study. (B) The experimental groups were asked to imagine zooming in

("left-right" or "zoom," as compared with standard) in either experiment.

Experiment 3a (left-right) For the "same" trials, a 2 (Trial Type: CC, WW) $\times 2$ (Instruction Set: standard, elaboration) mixed-design ANOVA revealed a main effect of trial type, $F(1,68)=5.95, p=.02, \eta^{2}=.08$, indicating stronger BE for $\mathrm{CC}$ than for WW trials. There were no main effect of instructions, $F(1,68)<1$, and no interaction, $F(1,68)<1$. For "different" trials, a 2 (Trial Type: CW, WC) $\times 2$ (Instruction Set: standard, elaboration) mixed-design ANOVA revealed a main effect of trial type, $F(1,68)=106.19, p<.001, \eta^{2}=.61$, indicating that participants gave larger ratings to $\mathrm{WC}$ than to $\mathrm{CW}$ changes from study to test, replicating previous work (e.g., Dickinson \& Intraub, 2008). No main effect of instructions emerged, $F(1,68)<1$, and no interaction, $F(1,68)<1$. Note that the absolute values of the $\mathrm{CW}$ and WC scores were used in the ANOVA, in order to compare participants' use of the scale (Dickinson \& Intraub, 2008).

Experiment 3b (zoom in/out) For the "same" trials, a 2 (Trial Type: CC, WW) $\times 3$ (Instruction Set: standard, elaboration zoom in, elaboration zoom out) mixed-design ANOVA revealed a marginal main effect of trial type, $F(1,46)=3.67$, $p=.06, \eta^{2}=.07$, indicating similar BE for CC and WW trials, with a trend toward more BE in CC than in WW. We observed no main effect of instructions, $F(2,46)=1.43, p=.25, \eta^{2}=$ .06 , and no interaction, $F(2,46)<1$. For "different" trials, a 2 (Trial Type: $\mathrm{CW}, \mathrm{WC}) \times 3$ (Instruction Set: standard, elaboration zoom in, elaboration zoom out) mixed-design ANOVA revealed a main effect of trial type, $F(1,46)=56.61, p<.001$, $\eta^{2}=.55$, indicating that participants gave larger ratings to WC than to $\mathrm{CW}$ changes from study to test, replicating previous work (e.g., Dickinson \& Intraub, 2008). There
B 口Standard $\square$ "Zoom in" $\square$ "Zoom out"

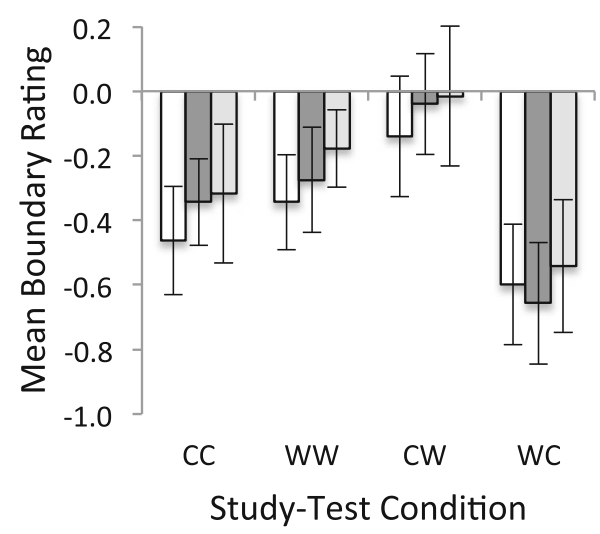

or zooming out (between participants). Negative ratings indicate that the test scene appeared to be closer than the original, and ratings of 0 indicate that the test scene appeared identical to the original. Error bars indicate $95 \%$ confidence intervals of the means. $\mathrm{C}=$ close-up, $\mathrm{W}=$ wide-angle

were no main effect of instructions, $F(2,46)<1$, and no interaction, $F(2,46)<1$.

\section{Object and spatial imagery}

We found individual differences in self-reported imagery ability in Experiment 3a, the study in which the OSIQ was included, with a significant difference between the top and bottom quartiles for the OSIQ object imagery and spatial imagery scores (see Table 1). In Experiment 3a (left-right), the top and bottom quartiles for object imagery were statistically different, $t(34)=15.42, p<.001, d=17.00$, and the same pattern held for spatial imagery, $t(34)=14.65, p<.001, d=12.63$. There was no difference between the CC scores for those in the top $(M=-0.42, S D=0.38)$ and bottom $(M=-0.38, S D=$ 0.35 ) quartiles for object imagery, $t(34)=0.34, p=.73, d=$ 0.11 . However, when CC scores were sorted by spatialimagery scores, we did find a difference between the $\mathrm{CC}$ scores for those in the top $(M=-0.55, S D=0.37)$ and those in the bottom $(M=-0.28, S D=0.26)$ quartile, $t(34)=2.52, p=$ $.02, d=0.84$. Participants who self-reported higher spatial imagery had larger (more negative) BE scores. In addition, a significant correlation emerged between spatial imagery and $\mathrm{CC}$ scores (higher imagery being associated with morenegative BE scores, $r=-.24, p<.05)$, but not between object imagery and $\mathrm{CC}$ scores $(r=-.08$, n.s. $)$.

\section{Discussion}

Robust BE was again observed for both $\mathrm{CC}$ and WW trials. Participants again gave larger ratings to $\mathrm{WC}$ than for $\mathrm{CW}$ trials, with participants responding "same" to $\mathrm{CW}$ trials on the rating scale. Once again, the instructions (standard or elaboration) did not affect BE scores. 
Unlike in Experiments 2a and 2b, which were challenged by relatively small sample sizes, in Experiment 3 a we observed a relationship between the spatial imagery subscale of the OSIQ and boundary extension, with self-reported strongspatial-imagery participants showing relatively large BE (the OSIQ was not included in Exp. 3b). By contrast, neither the VVIQ (Exp. 1b) nor the object subscale of the OSIQ (Exp. $3 a)$, both of which assess pictorial memory, were significantly related to BE scores. This contrasting pattern of spatial imagery being related to $\mathrm{BE}$ and pictorial imagery not being related to it makes intuitive sense, in that BE is spatial in nature. Future studies of BE should explore this further to clarify the role of individual differences in $\mathrm{BE}$ and why those may occur. Such an understanding would further inform the mechanisms that underlie BE itself.

\section{General discussion}

In the present series of six experiments, we examined whether various kinds of elaborative imagination enhance BE. Inspired by the proposal that $\mathrm{BE}$ results from a combination of scene representation being multisourced (Intraub, 2010; Intraub \& Dickinson, 2008) and subsequent source memory errors (e.g., Johnson et al. 1993), we developed study conditions to parallel elaborations that have been known to increase source memory errors for actions (Thomas et al. 2003). We asked participants to imagine additional sensory information while they were trying to remember photographs. Regardless of whether we asked them to imagine additional sounds, odors, tastes, or even visually what more of the photograph would look like, elaboration did not increase BE. Elaboration also did not reduce BE, which might have occurred if the act of generating additional information improved accuracy, as Foley et al. (2010) had found within the Deese/Roediger-McDermott paradigm for word and picture lists.

We found significant BE effects in all six experiments. Two key markers of BE showed up in every experiment - namely, that (a) CC trials and WW trials yielded BE, and (b) WC trials showed more BE than CW trials. Surprisingly, neither of these patterns was affected by cross-modal elaboration (Exps. 1a, $1 \mathrm{~b}, 2 \mathrm{a}$, and $2 \mathrm{~b}$ ) or visual elaboration (Exps. 3a and 3b). Our results were consistent across both RSVP (Exps. 2a and 2b) and blocked (the four other experiments) BE methods. The same patterns were reported with relatively short study periods (6 $\mathrm{s}$ in Exp. 1a) and relatively long study periods (15 s in Exp. 1b), and with both within-participants (Exps. 2a and 2b) and between-participants (Exps. 1a, 1b, 3a, and 3b) manipulations of instruction conditions. In short, with a variety of procedures, all of which resulted in clear BE, we found no evidence that elaboration had any effect on BE. BE may well be a source memory error, but explicit imagination does not appear to contribute to the misremembered boundaries of the originally presented visual stimulus.

Our experiments do tell us some interesting things about BE. In order to allow for substantial elaboration, we used photographs that were much more varied (in terms of the number of objects presented and wide-angle landscape views) than the typical BE photographs. With these photographs, participants had significant BE even for WW trials (which often show accurate performance; Exps. 1a, 1b, 3a, and 3b show this most clearly). Participants still gave larger ratings to WC than to $\mathrm{CW}$ trials, but with the world set of photographs, even CW trials were rated as being closer (Exps. 1a and $1 \mathrm{~b})$. Finding robust BE for a very different range of photographs highlights that $\mathrm{BE}$ is not limited to photographs with single, central objects. Experiments $2 \mathrm{a}$ and $2 \mathrm{~b}$ actually allowed for a comparison of the world pictures used in most of our experiments with more typical BE pictures, with no difference emerging in how participants rated the pictures. Scenes can be quickly classified in a variety of ways, including by structural properties such as openness, constancy properties such as temperature, and functional properties such as navigability (e.g., Greene \& Oliva, 2009a, b). Future research could examine whether these properties influence BE, though the present experiments suggest that structural and functional properties would be more likely to influence BE than constancy properties, given the correlation with spatial, but not object, imagery.

Within the multisource model of spatial representation, amodal completion is included as a contributor to $\mathrm{BE}$ (Intraub, 2010, 2012), and the strength of this contribution has been recently highlighted using very abstract scenes (Hale, Brown, McDunn, \& Siddiqui, 2015; McDunn, Siddiqui, \& Brown, 2014). In fact, an occluded edge is not enough to highlight the limited scope of a view (McDunn et al. 2014), and Hale et al. had to present extremal edges to stop participants from extending the boundaries of scenes of random abstract polygons on a field of random dots. Even with very abstract scenes - indeed, scenes with only surfaces and no objects - participants misremembered information from amodal completion as to what had been seen, showing source confusion, in support of BE as a source memory error (Hale et al. 2015; McDunn et al. 2014). The range of scenes across which BE is observed is quite broad, from the abstract shapes of Hale et al. and McDunn et al. to the rich world pictures of cities and markets in the present work.

We generally found more BE for the blocked procedure, and in particular the CW trials showed negative ("closer") ratings (Exps. 1a, 1b, 3a, and 3b) so that often they were correctly identified as being wider at test (as in Exps. 2a and $2 \mathrm{~b})$. In the RSVP procedure, participants are asked to remember three or four specific photographs for a much shorter duration of time, which might contribute to this different pattern for $\mathrm{CW}$ trials than with the blocked procedure. Dickinson and 
Intraub (2008) did vary the study time in an RSVP procedure, but with a much smaller range $(42-1,000 \mathrm{~ms})$, and found no effect of study time on BE. Regardless of the procedure, BE patterns were observed, and elaboration did not influence the $\mathrm{BE}$ ratings.

We also explored whether participants' self-reported visual imagery abilities correlated with their BE scores. Using the VVIQ, we found no relationship between pictorial imagery scores and BE (Exp. 1b). Using the OSIQ, we only found a significant correlation between BE scores and the spatial imagery subscore (Exp. 3a). However, we found no correlation with OSIQ and BE in Experiments $2 \mathrm{a}$ and $2 \mathrm{~b}$ - which is perhaps not surprising, given the relatively low $n$ in those withinparticipants experiments. It is important to note that participants did self-report a range of imagery ability and facility; these ratings just were not related to their BE scores. The only case in the present series of experiments in which a significant correlation occurred was when the elaboration condition was visuospatial ("imagine what would be to the left and right"). This is an intriguing new finding to explore within the $\mathrm{BE}$ literature.

Imagination, particularly rich sensory details, increases source memory errors for actions (Thomas et al. 2003). However, the act of imagining isolated objects improves memory, reducing source memory errors (Foley et al. 2010). Being specifically asked to elaborate either cross-modally (Exps. 1a, 1b, 2a, and 2b), or simply to imagine more visual input (Exps. 3a and 3b), led to no change in BE scores. Foley (2012) distinguished between spontaneous and intentional imagery; she argued that the latter allows participants to use records of cognitive operations to avoid false recognition (source memory) errors. In the present series of experiments, visual information was critical to deciding whether the angle of a test picture was the same, closer, or wider than its studyphase counterpart. By contrast, imagined sounds, smells, or tastes would be the same, regardless of visual angle. Experiments 1a, 1b, 2a, and $2 \mathrm{~b}$ focused on cross-modal elaboration ("imagine odors and sounds, also tastes" in Exp. 2b), so any visual imaginings would have been spontaneous. In Experiments $3 \mathrm{a}$ and $3 \mathrm{~b}$, by contrast, elaboration condition participants were instructed to imagine what was left or right of the shown scenes ( $3 \mathrm{a}$ ) or to imagine zoomed-in or zoomedout versions of the photographs ( $3 b$ ), so visual imaginings were intentional. Importantly, neither type of imagining resulted in a reduction of $\mathrm{BE}$ as compared with the standardinstruction conditions. We see this as a strength of this series of experiments, since it added generalizability to the conditions tested and failed to show modulation of the size of BE.

We fully acknowledge that null results are difficult to interpret, yet we used a wide range of methods (e.g., within- and between-participants designs, blocked and RSVP presentation, cross-modal and visual elaboration, short and long study times, world and object pictures, and spontaneous and intentional visual imagery) and failed to find an effect of elaboration instructions that have been effective in other source memory tasks (e.g., Foley, 2012; Foley et al. 2010; Thomas et al. 2003). At this point, we can only say that we do not have evidence to support the idea that explicitly imagined details can be misconstrued as visual information from a scene, and thus contribute to BE.

However, we also focused our investigation on the effect of elaborative imagery on BE. Future work should draw from other conditions that have been shown to increase or decrease source memory confusions (for a review, see Lindsay, 2008). For example, consider the Deese-Roediger-McDermott paradigm, in which participants misremember lures as being part of the original list. When participants are reminded of all possible sources with options on a memory test (e.g., heard the word, did not hear the word, did not hear the word but selfgenerated it, and both heard and self-generated it), source misattributions are reduced (Multhaup \& Conner, 2002, Exp. 2b; see Multhaup, 1995, for a similar pattern with the false-fame task). Moreover, Multhaup and Conner's participants were more likely to identify critical nonpresented lures as not heard but generated than as heard and generated, whereas they were more likely to identify the critical presented words as heard and generated than as not heard but generated. This pattern is consistent with participants evaluating information regarding auditory and cognitive operations as they assign the sources of test items. Exploring BE with an analogous set of test options that reminded participants of multiple possible sources on each trial (e.g., picture identical to one studied, picture closer than one studied, picture further away than one studied, picture identical to one studied but seems closer because I imagined what was beyond the original picture, picture identical to one studied by seems farther away because I did a mental close-up of the details of the original picture) may yield less BE than standard instructions. Identifying which, if any, manipulations affect $\mathrm{BE}$ will further our understanding of the extent to which $\mathrm{BE}$ is a source memory error.

An additional clue about the driving force behind $\mathrm{BE}$ might be drawn from Intraub et al. (2008), who showed that divided attention increases BE. When participants were asked to perform a difficult visual search task superimposed over a photograph, increased BE for the photograph was observed (Intraub et al. 2008). The researchers argued that the divided attention made it more difficult for participants to identify at test what had been amodally completed. In a similar vein, we have preliminary data suggesting that hand position, which affects the focus of attention (Abrams, Davoli, Du, Knapp, \& Paull, 2008; Davoli \& Abrams, 2009; Davoli, Brockmole, Du, \& Abrams, 2012; Davoli, Brockmole, \& Goujon, 2012; Davoli, Du, Montana, Garverick, \& Abrams, 2010), also affects the size of BE. Whereas divided attention increases BE because participants misattribute amodally completed details 
to the original scene (Intraub et al. 2008), increased attention from hand placement seems to reduce BE.

Yet another approach to BE is to focus on the spatial nature of the task. There is some evidence that spatial awareness, as measured by BE, is quite separate from memory (Kim, Dede, Hopkins, \& Squire, 2015). Patients with lesions limited to the hippocampus demonstrated as much $\mathrm{BE}$ as controls in both a drawing task and an RSVP task. Both groups were also sensitive to instructions regarding the likelihood of pictures in the RSVP task actually being the same. By contrast, patients' drawings were rated as being less accurate than controls' drawings were, and participants had lower recognition scores for the names of objects in the scenes than did controls. In a further experiment, patients with hippocampal damage included fewer sensory descriptions when asked to imagine what would be just out of view, but they provided spatial information similar to that of controls. Patients also had lower recall scores for the scenes they had been asked to extend. Kim et al. argued that the hippocampus is critical for memory, but damage to this region does not affect spatial awareness; thus, patients with hippocampus damage show impaired memory but preserved $\mathrm{BE}$. The spatial nature of $\mathrm{BE}$ is also supported by Intraub et al. (1998) finding that BE is influenced in healthy young adults by instructions to imagine specific spatial backgrounds, and by the present report of a positive correlation between the spatial subscale of the OSIQ and BE (Exp. 3a).

In summary, with six experiments that covered a range of design manipulations, we found no evidence that elaborative imagination affects the size of BE. We did, however, find a hint that spatial imagery ability may lead to greater BE. Further exploration of such individual differences and the roles of source-memory, attentional, and spatial processes in $\mathrm{BE}$ will be instructive in explaining why $\mathrm{BE}$ occurs.

Author note We thank the 2011-2014 Memory Lab and Perception \& Attention Lab students for help with data collection. Special thanks to Katherine Ness and Zach Stergar for helping develop the stimuli. Finally, we thank three reviewers and Jessica J. Good for helpful comments on the manuscript.

\section{References}

Abrams, R. A., Davoli, C. C., Du, F., Knapp, W., \& Paull, D. (2008). Altered vision near the hands. Cognition, 107, 1035-1047. doi:10. 1016/j.cognition.2007.09.006

Blajenkova, O., Kozhevnikov, M., \& Motes, M. A. (2006). Object-spatial imagery: A new self-report imagery questionnaire. Applied Cognitive Psychology, 20, 239-263. doi:10.1002/acp.1182

Davoli, C. C., \& Abrams, R. A. (2009). Reaching out with the imagination. Psychological Science, 20, 293-295. doi:10.1111/j.1467-9280. 2009.02293.x

Davoli, C. C., Brockmole, J. R., Du, F., \& Abrams, R. A. (2012a). Switching between global and local scopes of attention is resisted near the hands. Visual Cognition, 20, 659-668. doi:10.1080/ 13506285.2012.683049

Davoli, C. C., Brockmole, J. R., \& Goujon, A. (2012b). A bias to detail: How hand position modulates visual learning and visual memory. Memory \& Cognition, 40, 352-359. doi:10.3758/ s13421-011-0147-3

Davoli, C. C., Du, F., Montana, J., Garverick, S., \& Abrams, R. A. (2010). When meaning matters, look but don't touch: The effects of posture on reading. Memory \& Cognition, 38, 555-562. doi:10.3758/MC. 38.5.555

Dickinson, C. A., \& Intraub, H. (2008). Transsaccadic representation of layout: What is the time course of boundary extension? Journal of Experimental Psychology: Human Perception and Performance, 34, 543-555. doi:10.1037/0096-1523.34.3.543

Finke, R. A., Johnson, M. K., \& Shyi, G. C.-W. (1988). Memory confusions for real and imagined completions of symmetrical visual patterns. Memory \& Cognition, 16, 133-137. doi:10.3758/ BF03213481

Foley, M. A. (2012). Imagery encoding and false recognition errors: Exploring boundary conditions of imagery's enhancing effects. Memory, 20, 700-716. doi:10.1080/09658211.2012.697172

Foley, M. A., Foy, J., Schlemmer, E., \& Belser-Ehrlich, J. (2010). Imagery encoding and false recognition errors: Examining the role of imagery process and imagery content on source misattributions. Memory, 18, 801-821. doi:10.1080/09658211.2010.509731

Gagnier, K. M., \& Intraub, H. (2012). When less is more: Line drawings lead to greater boundary extension than do colour photographs. Visual Cognition, 20, 815-824. doi:10.1080/13506285.2012. 703705

Greene, M. R., \& Oliva, A. (2009a). The briefest of glances: The time course of natural scene understanding. Psychological Science, 20, 464-472. doi:10.1111/j.1467-9280.2009.02316.x

Greene, M. R., \& Oliva, A. (2009b). Recognition of natural scenes from global properties: Seeing the forest without representing the trees. Cognitive Psychology, 58, 137-176. doi:10.1016/j.cogpsych.2008. 06.001

Hale, R. G., Brown, J. M., McDunn, B. A., \& Siddiqui, A. P. (2015). An influence of extremal edges on boundary extension. Psychonomic Bulletin \& Review, 22, 961-966. doi:10. 3758/s13423-014-0751-x

Hubbard, T. L., Hutchison, J. L., \& Courtney, J. R. (2010). Boundary extension: Findings and theories. The Quarterly Journal of Experimental Psychology, 63, 1467-1494. doi:10.1080/ 17470210903511236

Intraub, H. (2004). Anticipatory spatial representation of 3D regions explored by sighted observers and a deaf-and-blind-observer. Cognition, 94, 19-37. doi:10.1016/j.cognition.2003.10.013

Intraub, H. (2010). Rethinking scene perception: A multisource model. In B. H. Ross (Ed.), Psychology of learning and motivation (pp. 231264). Burlington: Academic Press.

Intraub, H. (2012). Rethinking visual scene perception. Wiley Interdisciplinary Reviews: Cognitive Science, 3, 117-127. doi:10. 1002/wcs.149

Intraub, H., Daniels, K. K., Horowitz, T. S., \& Wolfe, J. M. (2008). Looking at scenes while searching for numbers: Dividing attention multiplies space. Perception \& Psychophysics, 70, 1337-1349. doi: 10.3758/PP.70.7.1337

Intraub, H., \& Dickinson, C. (2008). False memory 1/20th of a second later: What the early onset of boundary extension reveals about perception. Psychological Science, 19, 1007-1014. doi:10.1111/j. 1467-9280.2008.02192.x

Intraub, H., Gottesman, C. V., \& Bills, A. J. (1998). Effects of perceiving and imagining scenes on memory for pictures. Journal of Experimental Psychology: Learning, Memory, and Cognition, 24, 186-201. doi:10.1037/0278-7393.24.1.186 
Intraub, H., \& Richardson, M. (1989). Wide-angle memories of close-up scenes. Journal of Experimental Psychology: Learning, Memory, and Cognition, 15, 179-187. doi:10.1037/0278- 7393.15.2.179

Johnson, M. K., Hashtroudi, S., \& Lindsay, D. S. (1993). Source monitoring. Psychological Bulletin, 114, 3-28. doi:10.1037/0033-2909. 114.1.3

Kim, S., Dede, A. O., Hopkins, R. O., \& Squire, L. R. (2015). Memory, scene construction, and the human hippocampus. PNAS Proceedings of the National Academy of Sciences of The United States of America, 112, 4767-4772. doi:10.1073/ pnas. 1503863112

Lindsay, D. S. (2008). Source monitoring. In J. H. Byrne \& H. L. III Roediger (Eds.), Learning and memory: A comprehensive reference. Vol. 2: Cognitive psychology of memory (pp. 325-347). Amsterdam: Elsevier. doi:10.1016/B978-012370509-9.00175-3

Marks, D. F. (1973). Visual imagery differences in the recall of pictures. British Journal of Psychology, 64, 17-24. doi:10.1111/j.2044-8295. 1973.tb01322.x

Markham, R., \& Hynes, L. (1993). The effect of vividness of imagery on reality monitoring. Journal of Mental Imagery, 17, 159-170.
McAvinue, L. P., \& Robertson, I. H. (2007). Measuring visual imagery ability: A review. Imagination, Cognition and Personality, 26, 191211. doi:10.2190/3515-8169-24J8-7157

McDunn, B. A., Siddiqui, A. P., \& Brown, J. M. (2014). Seeking the boundary of boundary extension. Psychonomic Bulletin \& Review, 21, 370-375. doi:10.3758/s13423-013-0494-0

Mullally, S. L., Intraub, H., \& Maguire, E. A. (2012). Attenuated boundary extension produces a paradoxical memory advantage in amnesic patients. Current Biology, 22, 261-268. doi:10.1016/j.cub.2012.01. 001

Multhaup, K. S. (1995). Aging, source, and decision criteria: When false fame errors do and do not occur. Psychology and Aging, 10, 492497. doi:10.1037/0882-7974.10.3.492

Multhaup, K. S., \& Conner, C. A. (2002). The effects of considering nonlist sources on the Deese-Roediger-McDermott memory illusion. Journal of Memory and Language, 47, 214-228. doi:10. 1016/S0749-596X(02)00007-4

Thomas, A. K., Bulevich, J. B., \& Loftus, E. F. (2003). Exploring the role of repetition and sensory elaboration in the imagination inflation effect. Memory \& Cognition, 31, 630-640. doi:10.3758/ BF03196103 